\title{
SALA DE AULA 4.0: TREINANDO UM ENGENHEIRO DE PROCESSOS EM UM AMBIENTE MULTIDISCIPLINAR
}

\section{CLASSROOM 4.0: TRAINING A PROCESS ENGINEER IN A MULTIDISCIPLINARY ENVIRONMENT}

A. Valdman ${ }^{1}$ and R. P. Tiradentes ${ }^{1}$

${ }^{1}$ Federal University of Rio de Janeiro, Chemical Engineering Department, Rio de Janeiro, Rio de Janeiro, Brasil

${ }^{*}$ Corresponding author. Federal University of Rio de Janeiro, Department of Chemical Engineering, Rio de Janeiro, Brazil, Phone: +55 21 3938-7315

e-mail addressl: avaldman@eq.ufrj.br (A. Valdman).

\begin{tabular}{l} 
A R T I C L E I N F O \\
\hline Article history: \\
Received 2019-10-03 \\
Accepted 2019-12-20 \\
Available online 2019-12-20 \\
p a l a vras - chave \\
Indústria 4.0 \\
Engenharia de Processos \\
Sala de aula invertida \\
Experiência industrial \\
ke yw or $d s$ \\
Industry 4.0 \\
Process engineering \\
Flipped classroom \\
Industrial experience \\
\end{tabular}

\begin{abstract}
A B S T R A C T
Nowadays, operational data and technology is the core of chemical and biochemical industries. This multidisciplinary environment evokes new teaching and learning issues concerning the modern engineer capabilities and how to gain experience on industrial based standards. The classroom 4.0 is an industrial experience, involving Problem Based Learning and flipped classroom strategies, based on a semi-industrial boiler plant with fieldbus instruments and an open data infrastructure. The server is connected to the institutional network, allowing students to analyze 150 process variables and to approach the complexity of an industrial decision making environment. The developed methodology merges cognitive and traditional learning techniques to stimulate creativity and the analyses of subjective key performance indicators, such as On Stream Factor and Overall Equipment Effectiveness.
\end{abstract}




\section{INTRODUÇÃO}

A indústria 4.0 passou de uma tendência futurista para uma realidade focada no âmbito digital (Herman et. al., 2015). A consultoria PwC (Geissbauer et al., 2016) estima que serão investidos 45 bilhões de dólares por ano até 2020 para a digitalização dos ativos da indústria química, onde o uso de sistemas de gerenciamento e análise de dados está se tornando cada vez mais presente, com softwares capazes de monitorar e analisar informações de forma integrada com outras instâncias computacionais em um ambiente big data (Rojko, 2017). Interligam-se o controle de processos e os sistemas de gestão das empresas, através de servidores remotos com acesso à internet (Samad et al, 2007). Um dos maiores desafios modernos da indústria é a digitalização de ponta a ponta de todos os ativos físicos, conectando diretamente todos os setores da cadeia de valor: partindo dos fornecedores, passando pela unidade fabril até chegar aos clientes (European Parliament, 2015). Empresas e países já enxergam esta perspectiva, denominada indústria 4.0, como uma estratégia nacional de crescimento e participação na cadeia global de produção (Blanchet et al., 2014; FIRJAN, 2016). Neste sentido, ao longo da história, universidades estão sempre estudando como aproximar os objetivos acadêmicos e as demandas do ambiente industrial (Gregory et al., 2016).

Segundo Fadeeva e Kirilov (2015), as metodologias aplicadas em cursos superiores de engenharia não incentivam o desenvolvimento da criatividade aplicada à resolução de problemas, habilidade esta inerente ao atual profissional no mercado de trabalho (Demartini et al., 2017). Abordagens didáticas modernas, tais como Living Labs (Leminen et al., 2015) e CDIO (Conceive-Design-Implement-Operate) (Crawley et al., 2007), vem sendo apresentadas como metodologias centradas no aprendiz e na criatividade (Iborra et al., 2014).

O objetivo deste trabalho é contribuir na aproximação academia-indústria, disponibilizando aos futuros profissionais da engenharia de processos recursos básicos da indústria 4.0 aplicados a processos químicos. Um software industrial de gerenciamento de dados foi configurado para uma caldeira semi-industrial, disponibilizando a análise de dados em tempo real ou a partir de dados históricos, bem como Interfaces Homem Máquina (IHM) local e virtual com acesso individualizado. Através de um ambiente análogo ao encontrado no atual cenário industrial, contextualizando a abordagem big data, o aluno é estimulado a utilizar os conceitos teóricos adquiridos ao longo do seu curso superior, para traduzir um conjunto de dados reais em fenômenos químicos e físicos, gerando assim indicadores que representam o funcionamento da planta.

\section{METODOLOGIA}

Este trabalho foi desenvolvido no Laboratório de Automação e Controle (LAC), da Escola de Química (LADEQ/EQ/UFRJ), onde se encontra uma caldeira flamotubular vertical de porte semi-industrial com capacidade de $500 \mathrm{~kg} / \mathrm{h}$ de vapor saturado. A Figura 1 apresenta as principais instâncias da planta, incluindo os equipamentos de instrumentação, automação e controle de processo. A monitoração e o controle da planta são feitos por meio de instrumentação híbrida (tecnologias 4 a $20 \mathrm{~mA}$ e Fieldbus
Foundation), interligadas ao servidor responsável pela infraestrutura de dados. Este servidor é conectado à rede ethernet da EQ/UFRJ, que pode ser acessada de qualquer outro dispositivo conectado na rede.

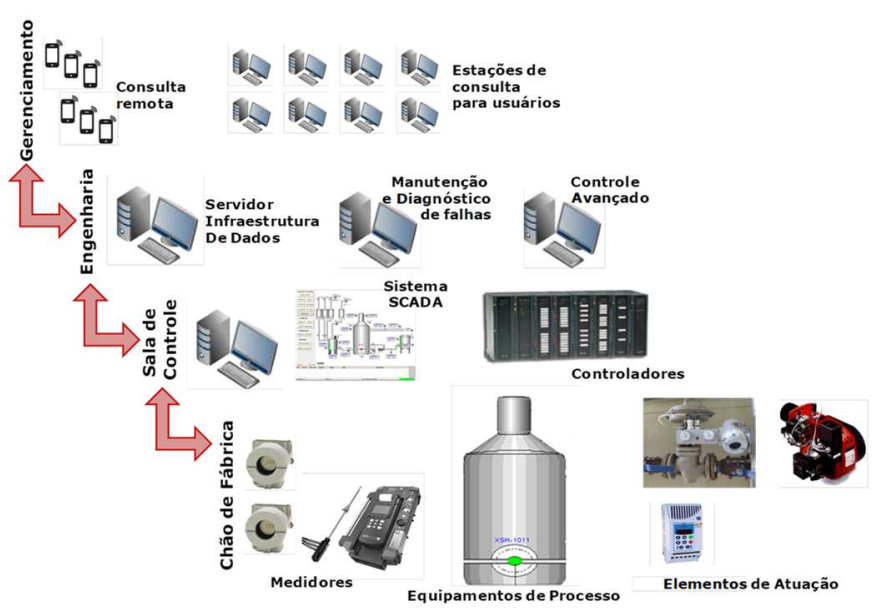

Figura 1 - Infra-estrutura industrial do processo.

O servidor de infraestrutura de dados é composto de um microcomputador com 2 GB de memória ram e processador Intel Core i3, utilizando como sistema operacional o Windows Server 2012, e pelos softwares PI System ${ }^{\circledR}$ da OSISOFT® e planilha eletrônica Microsoft Excel®. Uma base de dados única foi configurada para coletar e armazenar as informações de processo, exercendo a função de administrador central big data. A sala de aula para atividades didáticas é composta por 10 microcomputadores, com sistema operacional Windows XP®, onde foram instalados os softwares PI System Explorer $®$, Microsoft Excel® e o add-in PI DataLink®.

\section{1 Árvore de ativos}

A árvore de ativos da planta (Figura 2) que representa a base de dados contém 32 ramos e 164 frutos, conectados a 2 eixos principais (Central de Utilidades e Central de Processos). O eixo Central de Processos não faz parte do escopo do presente trabalho. Os eixos foram desenvolvidos de forma orientada ao processo, onde os primeiros ramos representam etapas e/ou equipamentos-chave (Combustão, Tanques, etc.), os ramos secundários representam equipamentos específicos (Tanque de água, Tanque de óleo, etc.) e os frutos, as variáveis de processo e propriedades físico-químicas. Até o presente momento, encontra-se disponível cerca de 2 anos de dados históricos armazenados, resultado de práticas experimentais oferecidas na caldeira aos cursos de graduação de Engenharia Química da EQ/UFRJ. São cerca de 150 variáveis (pontos analógicos e pontos discretos) com uma taxa de amostragem de $1 \mathrm{~s}$, correspondendo a mais de $170 \mathrm{MB}$ de memória.

No intuito de representar um ambiente industrial, foi desenvolvida uma interface homem máquina (IHM) local, com acesso a partir da sala de controle, e uma IHM virtual (Figura 3) com acesso online individualizado de qualquer dispositivo, móvel ou fixo, conectado à rede da Escola de Química através de navegador de internet. Sendo assim, cada usuário remoto tem acesso ao sinótico em tempo real do processo e à árvore de ativos do servidor central, podendo ainda desenvolver e 
compartilhar suas próprias telas de acesso remoto com outros usuários.

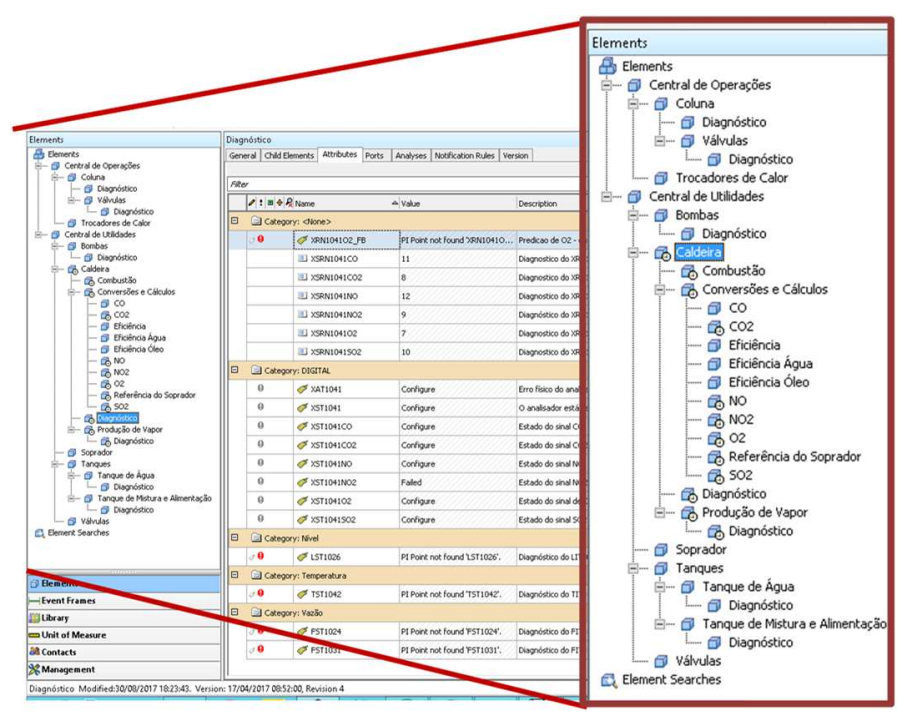

Figura 2 - Árvores de ativos.

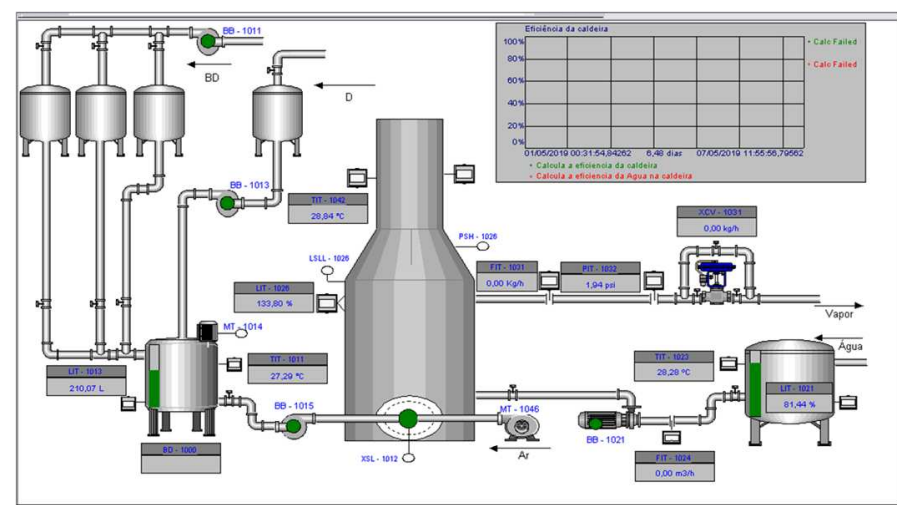

Figura 3 - Interface homem máquina do processo.

\subsection{Compactação de dados}

Uma etapa de configuração decisiva de um sistema de infraestrutura de dados é a parametrização do algoritmo de compactação de dados históricos. Em função do grande número de dados disponibilizados em tempo real, estes softwares oferecem mecanismos de exceção e compressão antes da etapa de armazenamento, que funcionam como um filtro dos dados coletados. No presente trabalho, as variáveis foram configuradas com um intervalo de coleta de 1s e a aplicação de 3 filtros: o Intervalo de Tempo, o Desvio de Exceção e o Desvio de Compressão. Basicamente, estes filtros são aplicados para descartar valores menores do que a tolerância em relação ao valor anterior dentro de um intervalo de tempo definido.

\subsection{Consulta de dados}

Os dados armazenados são consultados através de softwares de planilha eletrônica. A partir da definição de uma janela temporal de consulta, o método de Dados Brutos retorna todos os valores de uma determinada variável efetivamente armazenados na janela especificada. Neste caso, o intervalo de tempo entre os valores apresentados pode não ser uniforme, em função da parametrização do algoritmo de compactação. Por outro lado, além da janela temporal, o método de Dados Tratados solicita um intervalo de amostragem, retornando valores uniformemente espaçados no tempo. Este método é o mais utilizado na indústria, pois facilita a manipulação de séries temporais. No entanto, neste caso, o valor em um instante t que havia sido descartado em função do algoritmo de compactação, é estimado através de uma interpolação em tempo, considerando os valores vizinhos válidos mais próximos do instante de tempo t original.

\subsection{Metodologia de ensino proposta}

A metodologia tradicional de ensino encontra-se centrada no aprendizado de conteúdos teóricos que permitem entender um fenômeno químico ou bioquímico em escala industrial. A metodologia proposta encontra-se centrada no aprendiz, onde suas experiências sensoriais e intelectuais são utilizadas para fazer uma análise crítica do problema. Esta abordagem tem se mostrado bastante promissora, pois cada aluno busca desenvolver o seu próprio potencial de aprendizado, além de ser convidado a trabalhar coletivamente na busca de uma solução. Estas atividades reforçam ainda a importância dos conteúdos teóricos, muitas vezes vistos com desconfiança por essa nova geração de estudantes, onde, segundo eles, todas as respostas são instantâneas e estão disponíveis para acesso imediato.

Na metodologia tradicional, conforme apresentado na Figura 4, a partir de conhecimento das ciências básicas e de conceito iniciais de digrama de processos e instrumentação, o aluno é conduzido à etapa de modelagem fenomenológica. Se analisados de forma isolada, o aprendizado de conceitos teórico de fenômenos dinâmicos, que se alteram em função do tempo, torna-se bastante subjetivo e o aluno encontra dificuldades em aproximar esta etapa de um caso real. É comum ainda, devido a dificuldade de acesso a uma infraestrutura industrial, os modelos fenomenológicos serem demonstrados utilizando-se através de um ambiente de simulação. Estas práticas virtuais são de extrema importância para agregar conhecimento teórico, mas não necessariamente conduzem a uma tomada de decisão em um cenário industrial.
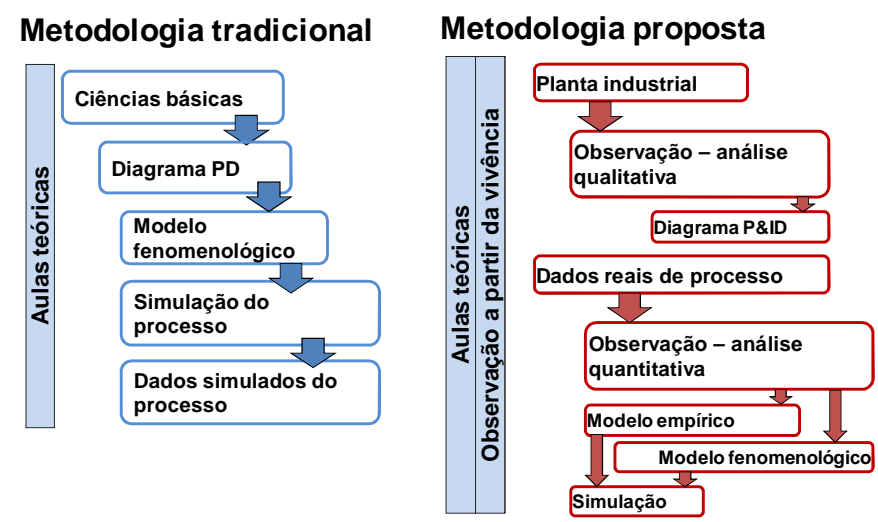

Figura 4 - Metodologia proposta. 
$\mathrm{Na}$ metodologia proposta, a primeira etapa de aprendizado é a imersão do aluno no ambiente industrial. Ao visitar a planta, o futuro engenheiro de processos tem acesso a um ponto de vista diferente, onde o reconhecimento do ambiente é feito a partir de informações teóricas adquiridas anteriormente e em uma análise cognitiva. Características visuais (dimensão, sujeira, pontos de corrosão localizados, disposição de equipamentos em um espaço físico, etc.), olfativas, auditivas (sons de bomba e compressores sendo ligados e desligados, sinalização de segurança, etc.) e táteis (superfícies mais quentes ou mais frias do que outras, com rugosidades diferentes, etc.) compõem alguns dos estímulos recebidos. A partir desta primeira análise, o aluno é conduzido a construir um diagrama de processos, incluindo a instrumentação envolvida. Ao longo deste trabalho, foi verificado que a introdução destas atividades para alunos de início de curso contribuem para a permanência e o estimulo do aluno nestes anos iniciais, onde o contato com as ciências básicas é bastante intenso e ocorre um distanciamento da vivência mais generalista e multidisciplinar da engenharia propriamente dita.

Somente após este primeiro contato com a realidade da planta, é fornecido o acesso aos dados reais da planta. A partir de então, as atividades são voltadas para análises mais quantitativas do processo, onde modelos empíricos são levantados e podem ser comparados com os modelos fenomenológicos desenvolvidos por simuladores. Atualmente, com a virtualização do aprendizado em várias instâncias, o aluno é convidado a se distanciar da realidade e da interação com o ambiente onde se encontra. $\mathrm{Na}$ área de engenharia de processos, um dos desafios introduzidos pela "era big data" é a transformação de dados em conhecimento fenomenológicos e não apenas em fenômenos estatísticos.

\section{RESULTADOS}

Após o desenvolvimento da estrutura, foi oferecida uma aula piloto, com uma duração total de $8 \mathrm{~h}$, para 16 alunos, cursando atualmente engenharia química ou química industrial em inicio ou final de curso, de diferentes universidades brasileiras. Durante a aula piloto, atividades cotidianas de um engenheiro de processos foram conduzidas, tais como:

a.Levantamento do processo a partir da planta, envolvendo atividades como o desenvolvimento do Diagrama P\&ID e a especificação de instrumento;

b.Consulta de histórico contendo dados de operação, envolvendo atividades de compactação e query de dados;

c. Transformação de números e dados em condições de processo e fenômenos químicos e bioquímicos,

d. Levantamento de indicadores de produtividade de um processo industrial.

Utilizando como exemplo o resultado fenomenológico de $8 \mathrm{~h}$ de operação da planta, foram propostas atividades que permitem a análise de alguns dos principais conceitos de uma plataforma big-data. Inicialmente, foi proposta uma avaliação da influência da etapa de compactação de dados no perfil dinâmico de uma variável de processo. Em seguida, considerando-se dados de histórico armazenados sem compactação, foi realizada uma análise da influência dos métodos de consulta de dados. Em função destas análises iniciais, foi feito um levantamento de algumas das funções de transferência do processo, contemplando conceitos de perturbação e modelagem empírica. Por fim, foram propostas discussões quantitativas e qualitativas sobre alguns indicadores de produtividade.

\subsection{Estrutura da aula}

A estrutura da aula foi dividida em duas categorias: o aprendizado conduzido pelo professor e a sala de aula invertida, onde o aluno resolve desafios propostos a partir da utilização das ferramentas e da participação ativa do mesmo em discussões técnicas. Dentre as atividades da primeira categoria, foram fornecidas informações básicas sobre os principais fenômenos físicos, químicos e bioquímicos que ocorrem na planta, integrando complementando informações teóricas obtidas em aulas tradicionais. Recursos básicos para interpretação de um diagrama típico de processos e instrumentação (diagrama P\&ID) também são apresentados, além de uma breve descrição das malhas de controle e intertravamentos de segurança da planta. A última etapa de aprendizado conduzido é a apresentação das ferramentas de software disponíveis e os conceitos básicos de infraestrutura de dados (árvore de ativos, IHM, compactação e consulta de dados). As atividades de sala de aula invertida são propostas ao longo do curso, incluindo atividades de construção da árvore de ativos; desenvolvimento de IHM específicas para acompanhamento da operação, da manutenção ou da produção; escolha de variáveis-chave para monitoramento; quantificação de parâmetros técnicos da operação; identificação de indicadores de produtividade. No presente trabalho são apresentadas as atividades envolvendo compactação e consulta de dados e propostas de análise dos indicadores de produtividade.

\subsection{Compactação de dados}

Considerando que a compactação de dados de processo influencia de forma distinta variáveis de natureza dinâmica rápida e lenta, foram selecionadas as variáveis de nível de água na caldeira (LT) e vazão volumétrica de alimentação de água à caldeira (FT) para esta etapa. Para efeitos de comparação, cada variável de processo foi configurada de forma duplicada na árvore de ativos: uma com os parâmetros de compactação de dados default (LTon e FTon) (intervalo de 10 minutos, Exceção de 0,1 e Compressão de 0,2) e outra (LToff e FToff), com os mesmos zerados. Testes de enchimento de água na caldeira, com a combustão desligada, foram conduzidos: a bomba de água foi mantida ligada durante por pelo menos 30 s e, em seguida, foi ligada e desligada em sequência, repetidas vezes. As quatro variáveis foram acompanhadas simultaneamente durante 9849 segundos, gerando um total de 9849 valores brutos coletados para cada variável.

Conforme apresentado na Tabela 1, o nível de água sem compactação apresentou o maior número de valores armazenados, pois o princípio de medição deste instrumento é a pressão, medida através de uma célula capacitiva, gerando oscilações naturais. Em analogia, menos pontos são armazenados para a vazão, pois a alimentação é constante, mantida através de uma bomba de deslocamento positivo. Verifica-se uma redução de $10 \%$ no armazenamento histórico de LToff devido a ocorrência de valores idênticos e consecutivos, descartados mesmo com os filtros de compactação desativados. No entanto, ao comparar o armazenamento da vazão sem e com compactação, percebe-se 
ainda uma redução de $73 \%$. Considerando-se que a vazão apresentou longos períodos em torno de um valor constante (ora com a bomba ligada ora com a bomba desligada), a maior redução percentual pode significar que a compactação é recomendada para esta variável, por não coletar valores temporais idênticos repetidos.

Tabela 1 - Análise da influência da compactação de dados de processo.

\begin{tabular}{|llll|}
\hline $\begin{array}{l}\text { Variável } \\
\text { LToff }\end{array}$ & $\begin{array}{l}\text { Compactação } \\
\text { ativada } \\
\text { (Sim/Não) }\end{array}$ & $\begin{array}{l}\text { Valores } \\
\text { armazenados }\end{array}$ & Redução (\%) \\
\hline NTão & 8907 & 10 \\
FToff & Sim & 497 & 95 \\
FTon & Não & 593 & 94 \\
& Sim & 158 & 98 \\
\hline
\end{tabular}

A Figura 5 representa uma consulta de Dados Brutos à vazão de água com e sem compactação durante 1 min. Na região 1, a vazão de água flutua em torno de um valor constante e, por isso, não há armazenamento de dados com a compactação ativa. Na região onde o perfil de vazão se altera rapidamente, todos os dados coletados são armazenados, com ou sem compactação, sobrepondo os pontos. Além dos conceitos de Indústria 4.0, esta etapa de análise permite ainda a discussão e visualização de conceitos estatísticos envolvidos, tais como variância, variabilidade e desvio padrão.

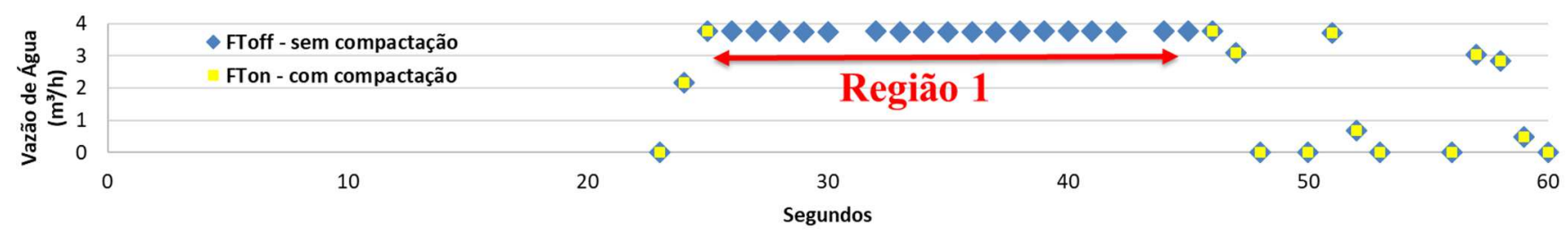

Figura 5 - Perfil dinâmico da vazão de água com e sem compactação.

\subsection{Consulta de dados}

A vazão de alimentação sem compactação (FToff) foi selecionada para analisar a influência do método de consulta de dados. A Figura 6 apresenta os perfis dos métodos de Dados Brutos e de Dados Tratados (com um intervalo de consulta de 1s ) no mesmo recorte de tempo utilizado na etapa anterior. $\mathrm{Na}$ região 1, ao longo da qual a variável flutua em torno de um valor constante, não aparecem diferenças significativas entre os métodos de consulta e os pontos brutos armazenados se sobrepõem aos dados interpolados em tempo. Na região posterior, onde a dinâmica é brusca e oscilatória, os resultados dos métodos de consulta não coincidem, sugerindo erros na interpretação. Os instantes de tempo onde as perturbações são detectadas diferem, além da resposta dinâmica em si. Foram estimuladas ainda discussões sobre ruído e precisão de instrumentos, relação entre número e fenômeno, valor online $\mathrm{x}$ valor histórico.

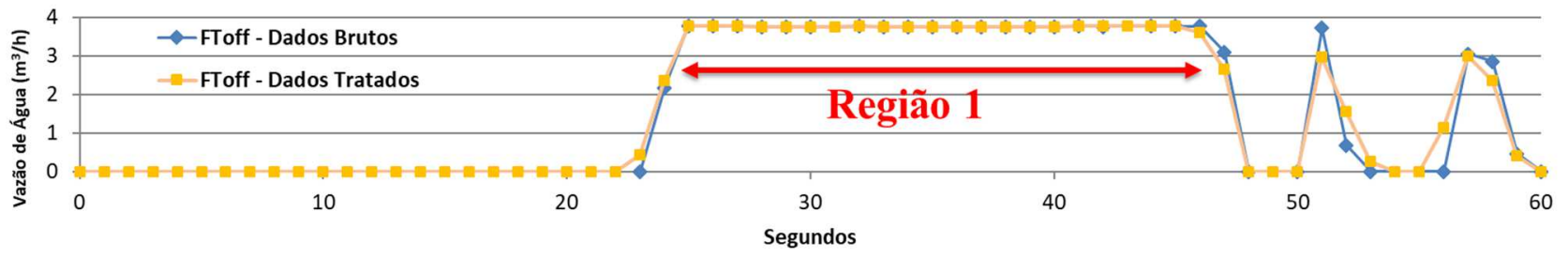

Figura 6 - Perfil dinâmico da vazão de água sem compactação, em função do método de consulta.

\subsection{Indicadores de produtividade}

Fundamentos de cálculos estatísticos são apresentados como subsídio aos alunos para solucionar os desafios propostos na sala de aula invertida, além de alguns recursos matemáticos oferecidos pelo software de infraestrutura de dados. A obtenção de índices de produtividades, ou KPIs, é feita a partir de uma análise subjetiva do comportamento das variáveis de processo e da utilização de médias, totalização e contagem de tempo associados às variáveis de processo historiadas. Os indicadores de produtividade propostos foram o On Stream Factor (OSF) índice de performance definido como a fração de tempo em que a unidade de processamento operou - e o Overall Equipment Effectiveness (OEE) - índice de produtividade que engloba conceitos de qualidade do produto, performance da planta e disponibilidade da unidade. Considerando ainda que existem industrialmente múltiplas formas de quantificar estes KPIs, nesta etapa os alunos são estimulados a defender e argumentar tecnicamente sobre como definir conceitualmente os mesmos e sobre a escolha das variáveis de processo selecionados para calculá-los, frente às múltiplas interpretações cabíveis.

\section{CONCLUSÃO}

A estrutura desenvolvida oferece ambiente adequado à exploração dos conceitos da indústria 4.0, permitindo em especial a análise in loco da influência dos mecanismos de compactação e consulta de dados em uma plataforma big data. 
Em processos industriais químicos e bioquímicos, onde é comum a conversão de séries temporais em informação fenomenológica, a etapa de consulta aos dados é decisiva. O estudo realizado observou capacidade de redução de até $70 \%$ na quantidade de pontos coletados com os recursos de compactação disponíveis. Entretanto, o perfil gerado pela consulta de dados tratados prejudica a análise do comportamento dinâmico do processo. A sala de aula 4.0 pode ser utilizada por até 20 alunos, com acesso simultâneo e individualizado de cada microcomputador ao servidor de infraestrutura de dados. $\mathrm{Na}$ aula piloto, $100 \%$ dos alunos afirmaram que a experiência acrescentou algo novo ao sua formação em engenharia de processos. Além disso, $40 \%$ e $60 \%$ dos alunos consideraram a abordagem boa e excelente, respectivamente. $\mathrm{O}$ desenvolvimento de cada tarefa independe da condução direta do professor, permitindo ao aluno explorar os recursos disponibilizados sem riscos de produção. A robusta arquitetura final é voltada para a melhor experiência e adequação do usuário final ao longo dos diferentes níveis de sua formação acadêmica. Este ambiente disponibiliza ainda um contexto de sala de aula invertida, onde o aluno tem liberdade criativa para desenvolver o seu lado profissional mais intuitivo, além de vivenciar os sucessos e os fracassos de suas escolhas técnicas a cada desafio técnico proposto.

\section{R E F E R E N C I A S}

BLANCHET, M.; RINN, T.; VON THARDEN, G.; THIEULLOY, G. de. Industry 4.0 The new industrial revolution how Europe will succeed. Think Act, 2014.

CRAWLEY, E. F., ÖSTLUND, S.; MALMQVIST, J.; BRODEUR D. R. Rethinking Engineering Education The CDIO Approach. Springer, 2007.

DEMARTINI, C.; BENUSSI, L. Do Web 4.0 And Industry 4.0 Imply Education X.0?, It Professional, v.19, n. 3, p. 47, jun. 2017.

EUROPEAN PARLIAMENT, Industry 4.0 Digitalization for productivity and growth, 2015.

FADEEVA, V. N.; KIRILLOV, N. P. The Issues of Development of a Creative Professional, Procedia Social and Behavioral Sciences, v.166, p. $333-338$, jan. 2015.

FIRJAN, Panorama da Inovação. Indústria 4.0. Publicações FIRJAN, 2016.

GEISSBAUER, R.; VEDSO, J.; SCHRAUF, S. Industry 4.0: Building the Digital Enterprise. Pwc, 2016.

GREGORY, P.; BARROCA, L.; SHARP, H.; DESHPANDE, A.; TAYLOR, K. The Challenges That Challenge: Engaging With Agile Practitioners Concerns, Information and Software Technology, v. 77, p.92-104, sep. 2016;

HERMANN, M.; PENTEK, T.; OTTO, B. Design Principles For Industrie 4.0 Scenarios: A Literature Review. In: 49TH HAWAII INTERNATIONAL CONFERENCE ON SYSTEM SCIENCES, 2016, Koloa, USA. Anais: IEEE Computer Society, p. 3928-3937.

IBORRA, M.; RAMÍREZ, E.; TEJERO, J.; BRINGUÉ, R.; FITÉ, C.; CUNILL, F. Revamping of teaching-learning methodologies in laboratory subjects of the Chemical Engineering undergraduate degree of the University of Barcelona for their adjustment to the Bologna process,
Education for Chemical Engineers, v.9, n. 3, p. e43e49, jul. 2014.

LEMINEN, S.; NYSTRÖMC, A. G.; WESTERLUNDD, M. A typology of creative consumers in living labs, Journal of Engineering and Technology Management, v.37, p. 6 -20 , jul/sep. 2015.

ROJKO, A., Industry 4.0 Concept. Background And Overview. I. J. Interactive Mobile Technologies, v.11, n. 5, p. 7790, 2017.

SAMAD, T.; MCLAUGHLIN, P.; LU, J. System Architecture For Process Automation: Review And Trends. Journal of Process Control, v.17, n. 3, p. 191-201, mar. 2007. 